\title{
TERAHERTZ IFEL/FEL MICROBUNCHING FOR PLASMA BEATWAVE ACCELERATORS
}

\author{
C. Sung, S. Ya. Tochitsky, J. Ralph, P. Musumeci, S. Reiche, \\ C. Clayton, J. B. Rosenzweig, C. Pellegrini, C. Joshi, \\ Neptune Laboratory, University of California, Los Angeles, CA 90095, U.S.A.
}

\begin{abstract}
In order to obtain monoenergetic acceleration of electrons, phase-locked injection using electron microbunches shorter than the accelerating structure is necessary. For a laser-driven plasma beatwave accelerator experiment, we propose to microbunch the electrons by interaction with terahertz $(\mathrm{THz})$ radiation in an undulator via two mechanisms- Free Electron Laser (FEL) and Inverse Free Electron Laser (IFEL). Since the high power FIR radiation will be generated via difference frequency mixing in GaAs by the same $\mathrm{CO}_{2}$ beatwave used to drive the plasma wave, electrons could be phase-locked and pre-bunched into a series of microbunches separated with the same periodicity. Here we examine the criteria for undulator design and present simulation results for both IFEL and FEL approaches. Using different $\mathrm{CO}_{2}$ laser lines, electrons can be microbunched with different periodicity $300-100 \mu \mathrm{m}$ suitable for injection into plasma densities in the range $10^{16}-10^{17} \mathrm{~cm}^{-3}$, respectively. The requirements on the $\mathrm{THz}$ radiation power and the electron beam qualities are also discussed.
\end{abstract}

\section{INTRODUCTION}

Due to the limitation of the energy gain gradient and cost efficiency of conventional RF-based accelerators, advanced accelerator concepts, such as laser or electron beam driven plasma wave based structures, have been proposed as potential solutions for the next generation accelerators and studied worldwide [1]. In recent years, proof-of principle experiments have shown gradients much higher than conventional accelerators [2] in a short distance. However, low quality of the electron beam produced by plasma-based accelerators, for example, the energy spread is continuous, practically limits acceleration to one stage. Therefore, we introduce a plasma LINAC concept in which the injected particles are bunched on a scale shorter than the wavelength of the accelerating structure and with the same periodicity such that monoenergetic acceleration of electrons can be obtained. For a plasma wave resonantly driven at plasma densities $10^{16}-10^{17} \mathrm{~cm}^{-3}$, the plasma wavelength $\lambda_{\mathrm{p}}$ is equal to $340-100 \mu \mathrm{m}$, respectively. In order to inject electrons into a narrow phase interval of the plasma wave, an electron beam needs to be prebunched into a series of $\sim 50-15 \mu \mathrm{m}$ long microbunches that separated by the plasma wavelength. It was demonstrated that IFEL method can be used at a wavelength as short as $10 \mu \mathrm{m}$ to produce a train of microbunches for a stiff relativistic electron beam [3]. The comb of $\mathrm{CO}_{2}$ laser lines is an ideal source for generating step tunable radiation in the FIR range of 100-1000 $\mu \mathrm{m}$ through difference frequency generation (DFG) in a nonlinear crystal. Thus there is a strong motivation both to develop a DFG-based source of $\mathrm{THz}$ radiation and to demonstrate microbunching for laser-plasma accelerators in the $10^{16}-10^{17} \mathrm{~cm}^{-3}$ density ranges.

For the $\mathrm{CO}_{2}$ laser driven Plasma Beatwave Accelerator (PBWA) experiment at the Neptune laboratory where $\lambda_{\mathrm{p}} \sim$ $340 \mu \mathrm{m}(\sim 1 \mathrm{THz})$, it was proposed to use a THz IFEL prebuncher for producing short, $50 \mu \mathrm{m}$ electron bunches phase-locked with the accelerating structure [4]. Phase locking between the electrons and PBWA becomes possible since the same laser beatwave is used to excite the relativistic plasma wave and to generate $\mathrm{THz}$ radiation via DFG to drive the IFEL prebuncher.

However, IFEL THz microbunching requires a MW power $\mathrm{THz}$ radiation [5]. Thus the IFEL microbuncher will be a single-shot experiment since high $\mathrm{CO}_{2}$ power will be required to generate $\mathrm{MW}$ level $\mathrm{THz}$ radiation. In this paper, we also consider a high-gain, single pass $\mathrm{THz}$ FEL microbunching experiment. The THz FEL microbunching requires $\mathrm{a} \sim 1 \mathrm{~kW}$ seed pulse which could generated using commercial $\mathrm{CO}_{2}$ lasers at $1 \mathrm{~Hz}$.

In this paper, the comparison between IFEL and FEL approaches for obtaining microbunched electrons is presented. Simulation results of detailed 3-D modeling of IFEL and FEL microbunching and optimization of the undulator design are shown.

\section{MICROBUNCHING EXPERIMENTS AT NEPTUNE LABORATORY}

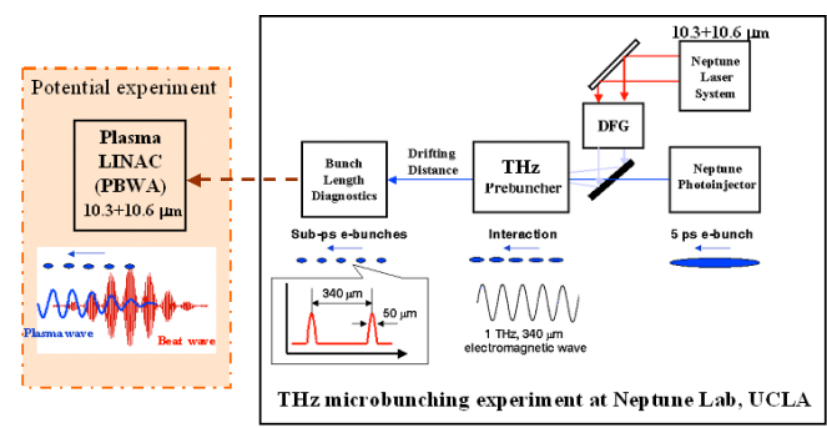

Figure 1. Schematic of $\mathrm{THz}$ microbunching experiments at Neptune Lab, UCLA

In the PBWA experiment at the Neptune Laboratory at UCLA, two lines of a $\mathrm{CO}_{2}$ laser $(10.6$ and $10.3 \mathrm{~m})$ were used to excite a relativistic plasma beat wave $\left(\lambda_{\mathrm{p}}=340\right.$ $\mu \mathrm{m})$ with an acceleration gradient $\sim 1-3 \mathrm{GeV} / \mathrm{m}$ [6]. Before the plasma LINAC experiment, we would like to 
study $\mathrm{THz}$ microbunching in order to choose the optimal technique. Both IFEL and FEL techniques must produce a modulated electron beam phase-synchronized with the $\mathrm{CO}_{2}$ laser beatwave. As shown in Fig. 1, a high-power, two-wavelength $\mathrm{CO}_{2}$ laser beam is sent into a nonlinear crystal (GaAs) for DFG. This newborn radiation will be focused into a planar $\mathrm{THz}$ prebuncher and sent collinearly with an relativistic electron beam coupled through a hole in a mirror.

The electron beam coming from a photoinjector will interact with the $\mathrm{THz}$ radiation via IFEL/FEL mechanism while going through the undulator and get bunched while drifting in free space. After drifting, the bunched beam will be analyzed using different bunch length diagnostics, such as CTR measurements, and a RF cavity deflector [7]. It is important that on the $\mathrm{THz}$ scale direct measurement of longitudinal dynamics of electron bunches is still possible.

\section{COMPARISON BETWEEN IFEL AND FEL MICROBUNCHING MECHANISMS}

When an electron beam wiggles along the axis of the undulator, the energy perturbation on each electron caused by a co-propagating electromagnetic (EM) wave is dependent on its initial phase within the EM wave. Such an energy-modulated beam can be compressed after drifting an optimal distance. If an electron experiences a full cycle of changing of electric field while wiggling one period in the undulator, the energy modulation is maximized and it is called the resonant condition:

$$
\lambda=\frac{\lambda_{w}}{2 \cdot \gamma^{2}} \cdot\left(1+\frac{K^{2}}{2}\right)
$$

, where $\mathrm{K}=\mathrm{eB}_{\mathrm{w}} / \mathrm{mc}^{2} \mathrm{k}_{\mathrm{w}}$ is the dimensionless undulator parameter, $\lambda$ is the radiation wavelength, $\gamma$ the electron energy, $\lambda_{\mathrm{w}}$ the undulator wavelength, $\mathrm{k}_{\mathrm{w}}$ the undulator wave number and $\mathrm{B}_{\mathrm{w}}$ the undulator magnetic field.

In a planar undulator with a focused laser beam, the energy perturbation of each electron is:

$$
\frac{\partial \gamma}{\partial z}=\frac{K}{\gamma} \cdot \frac{k K_{L}}{\sqrt{1+\frac{\left(z-z_{w}\right)^{2}}{Z_{r}^{2}}}} \cdot \frac{J J(K)}{2} \sin (\psi)
$$

where $\mathrm{K}_{\mathrm{L}}$ is the radiation dimensionless parameter $\left(\mathrm{eE}_{\mathrm{o}} / \mathrm{mc}^{2} \mathrm{k}\right), \psi$ is the phase of coupling between the wiggling motion and the EM wave $\left(\psi=\left(\mathrm{k}+\mathrm{k}_{\mathrm{w}}\right) \mathrm{z}-\omega \mathrm{t}\right)$, and $J J$ is the Bessel factor due to the planar geometry. Those equations show that for a given electron energy $\gamma$ and same radiation power, the larger the $\mathrm{K}$ of the undulator, the stronger the energy modulation is imposed on the electrons.

Besides the energy perturbation due to the EM wave, wiggling electrons also radiate coherently to amplify the EM wave. In the FEL, when bunching occurs, the radiation grows and consequently bunches the electrons more. Eventually, the EM wave power reaches saturation. This occurs when electrons lose too much energy and are no longer resonant. The net energy exchange is from the e-beam to the EM wave. In the FEL microbunching, the e-beam qualities are important since the e-beam plays a major role in the positive feedback mechanism. Whether the initial radiation power level exceeds the saturation power decides either IFEL or FEL mechanism dominates the microbunching process. In the IFEL, the initial power level is high such that the newborn radiation is negligible and the net energy exchange is from the EM wave to the electron beam. Therefore, in the IFEL microbunching, electrons are merely test particles.

\section{IFEL MICROBUNCHING}

We use a 3D code "TREDI", which calculates the Lorenz force applied on each electron and calculates its position and energy along time, to model the IFEL bunching effect. We consider two optical schemes --. diffraction-dominated and guiding in a hollow waveguide--- with parameters $\lambda=340 \mu \mathrm{m}, \gamma=20$ and energy spread $0.25 \%$. In the simulations, a focused Gaussian $\mathrm{THz}$ beam is used for the diffraction-dominated case and for the waveguide case a planar wave approximation is used.

In the diffraction dominated case [5], we chose a $50 \mathrm{~cm}$ long undulator with $\lambda_{\mathrm{w}}=9 \mathrm{~cm}, \mathrm{~B}_{\mathrm{w}}=0.24 \mathrm{~T}(\mathrm{~K}=2)$ and $4 \mathrm{~cm}$ gap as an example. The gap between the magnets is chosen to accommodate the whole FIR beam inside the entire length of the undulator. We found that with FIR power around $10 \mathrm{MW}$ when the Rayleigh range is equal to the undulator length, $40 \%$ particles are bunched in $45 \mu \mathrm{m}$ (FWHM) at the optimal drifting distance of $1.6 \mathrm{~m}$.

In the waveguide case, a $40 \mathrm{~cm}$ long undulator with $\lambda_{\mathrm{w}}=5 \mathrm{~cm}, \mathrm{~B}_{\mathrm{w}}=0.66 \mathrm{~T}(\mathrm{~K}=3)$ and $1 \mathrm{~cm}$ gap is used. The waveguide inner diameter (ID) is chosen to be $8 \mathrm{~mm}$ such that the propagating mode size can cover the whole wiggling e-beam inside the waveguide. With $1 \mathrm{MW} \mathrm{THz}$ power, $50 \%$ particles are bunched into a series of $50 \mathrm{~m}$ microbunches after $1 \mathrm{~m}$ drifting.

Further optimization could be done to lower the $\mathrm{THz}$ power requirement, however, when the initial $\mathrm{THz}$ power is too low, the radiation generated by the perturbed ebeam couldn't be neglected. Therefore it's beyond the capability of TREDI code and using an FEL code is required.

\section{FEL MICROBUNCHING}

It is known that radiation power increase in a high-gain, seeded FEL is directly related to the microbunching process occurring along the undulator. As seen in Eq. 1, by tuning energy $\gamma$ of electron beam in a small range, one can match the prospective resonant wavelengths in a given undulator. Considering the $\mathrm{THz}$ wavelength range and the Neptune photoinjector operating range, we choose the resonant condition (solid curve in Fig.4) such that $\gamma=26$ and 15 corresponds to $\lambda=100 \mu \mathrm{m}$ and $300 \mu \mathrm{m}$, respectively. As seen in Fig. 4, to maximize the bunching effect, $\lambda_{\mathrm{w}}$ and $\mathrm{B}_{\mathrm{w}}$ are chosen along the solid cure for the largest possible $\mathrm{K}$ (dashed lines). However, the Halbach formula (dotted lines) limits the choices because of the physical restriction in building magnets with small 
periods. Since the seed power and interaction is weaker in the FEL, guiding of the $\mathrm{THz}$ radiation is the only realistic option for a meter or longer undulator. After the optimization, we ended up with a planar undulator with $\lambda_{\mathrm{w}}=2.7 \mathrm{~cm}$, and $\mathrm{B}_{\mathrm{w}}=1.14 \mathrm{~T}(\mathrm{~K}=2.85)$ and the waveguide ID is chosen to be $5 \mathrm{~mm}$. Note that the mode size of $\mathrm{THz}$ radiation inside the waveguide is around $1.5 \mathrm{~mm}$. It covers the whole wiggling motion amplitude $(<700 \mu \mathrm{m})$ plus the electron beam size $\left(\sigma_{\text {r.m.s }}<220 \mu \mathrm{m}\right.$. $)$.

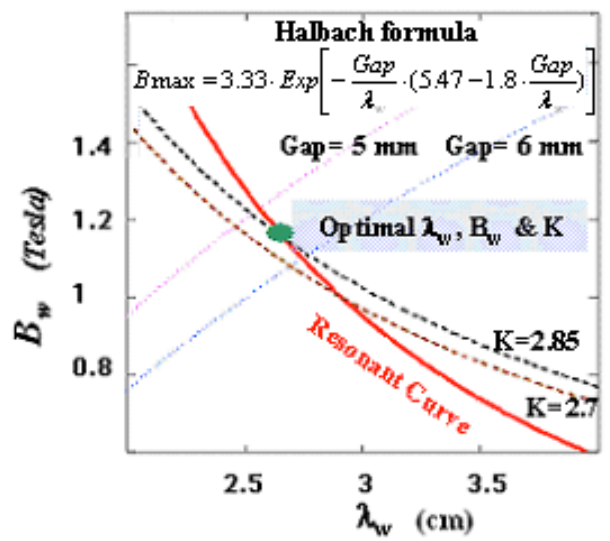

Figure 2: Optimization of undulator parameters for waveguide FEL microbunching

A 3-D, time-dependent code "Genesis" used for modeling the microbunching process includes the space charge effect. For FEL interactions, the most critical factor is the e-beam current: the larger the beam current, the faster the electrons are bunched. Using a $1.8 \mathrm{~m}$ long undulator with parameters presented in Table 1, an energy modulation $\Delta \gamma / \gamma \sim 0.5 \%$, sufficient for microbunching can be obtained with a modest 40 A peak current $(\sim 0.5 \mathrm{nC})$ and a $1 \mathrm{~kW}$ seed pulse at $200 \mu \mathrm{m}$ (Fig. 5c). We consider using an additional dispersive magnet after the undulator in order to shorten the drifting distance. Note that the resonant $\gamma$ is increased to 19.5 in comparison with free space propagation to match the phase velocity of $\mathrm{THz}$ radiation in the waveguide.

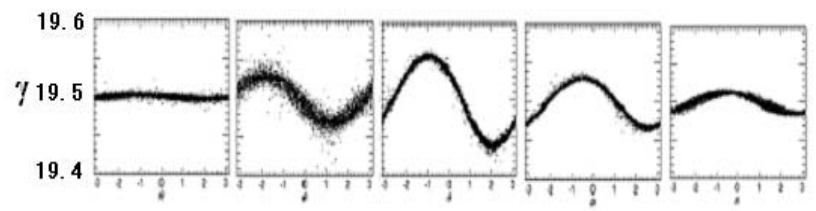

(a) $-2 \mathrm{~mm}$ (b)center (c) $+1.7 \mathrm{~mm}$ (d) $+4 \mathrm{~mm}$ (e) $+6 \mathrm{~mm}$ Figure 3: Phase space distribution at different slices after the e-beam propagates $1.8 \mathrm{~m}$ inside the $\mathrm{K}=2.85$ undulator

As seen in Fig. 3, the energy modulations at different slices are different. That is caused by a slippage effect when number of periods of the $\mathrm{THz}$ wave contained in the electron pulse is smaller than number of wiggling periods. Due to the slippage between the e-beam and the amplified $\mathrm{THz}$ radiation, electrons constantly "see" the radiation amplified by the other electrons behind them because the newborn radiation always overtakes the electrons that generate it. Therefore, the energy modulation is always stronger for the electrons slightly before the center of the Gaussian pulse. This phenomenon results in inhomogeneous modulation of the electron beam.

\begin{tabular}{|c|c|c|c|}
\hline \multicolumn{4}{|c|}{ Table 1: Parameters for Neptune waveguide (ID=5mm) } \\
FEL microbunching experiment \\
\hline \multicolumn{2}{|c|}{ E-beam parameters } & \multicolumn{2}{c|}{ THz radiation parameters } \\
\hline Energy & $8-14 \mathrm{MeV}$ & $\begin{array}{c}\text { Wavelengt } \\
\text { h }\end{array}$ & $100-300 \mu \mathrm{m}$ \\
\hline Pulse length & $\begin{array}{c}<5 \mathrm{ps} \text { r.m.s. } \\
(12 \text { ps FWHM) }\end{array}$ & $\begin{array}{c}\text { Pulse } \\
\text { length }\end{array}$ & $\sim 200 \mathrm{~ns}$ \\
\hline Current & $20-60 \mathrm{~A}$ & Seed power & $1-2 \mathrm{~kW}$ \\
\hline Beam size & $120-220 \mu \mathrm{m}$ & Intensity & $>10 \mathrm{~kW} / \mathrm{cm}^{2}$ \\
\hline $\begin{array}{c}\text { Transverse } \\
\text { emittance }\end{array}$ & $5-15 \mathrm{~mm}-\mathrm{mrad}$ & Rep. rate & $0.25-1 \mathrm{~Hz}$ \\
\hline \multicolumn{4}{|c|}{ Undulator parameters } \\
\hline Period & $2.7 \mathrm{~cm}$ & Gap & $5.5 \mathrm{~mm}$ \\
\hline Strength & $1.14 \mathrm{~T}$ & Length & $1.8 \mathrm{~m}$ \\
\hline $\mathrm{K}$ & 2.85 & $\begin{array}{c}\text { Symmetric and } \\
\text { constant focusing }\end{array}$ \\
\hline
\end{tabular}

\section{STATUS AND FUTURE PLANS}

In this paper, we show both IFEL and FEL approaches are suitable for bunching electrons in a $\mathrm{THz}$ scale. In the Neptune lab, a waveguide FEL experiment using a $1.8 \mathrm{~m}$ long undulator is in progress. We plan to study the longitudinal dynamics of the FEL microbunching with 1 $\mathrm{Hz}, 1 \mathrm{~kW}$ seed source. Since the optimization of IFEL and FEL undulator parameters is the same except the length, $60 \mathrm{~cm}$ long section of that undulator will also be used to study IFEL microbunching, which produces a uniformly modulated electron beam.

Work supported by the U.S. Department of Energy under Contract No. DE-FG03-92ER40727.

\section{REFERENCES}

[1] T. Tajima and J. M. Dawson, Phys. Rev. Lett., vol. 43, pp. 267-270 (1979)

[2] E. Esarey, P. Sprangle, J. Krall, and A. Ting, IEEE Trans. on Plasma Science, vol. 24, pp. 252-288 (1996)

[3] W. D. Kimura, M. Babzien, I. Ben-Zvi, et al, Phys. Rev. Lett., vol. 92, 054801-1-054801-4

[4] S. Ya. Tochitsky, P. Musumeci, C. E. Clayton, et al, AAC Proceeding 2002, pp. 786-795

[5] C. Sung, S. Ya. Tochitsky, P. Musumeci, et al, AAC Proceeding 2004, pp. 922-928

[6] S. Ya. Tochitsky, R. Narang, C. V. Filip, et al. Phys. Rev. Lett., vol. 92, 095004-1-095004-4 (2004)

[7] R. J. England, D. Alesini, A.Doyuran, et al, AAC Proceeding 2004, pp. 414-420 